\title{
Catalogue of selected experimental plans
}

\author{
Małgorzata Graczyk, Bronisław Ceranka \\ Department of Mathematical and Statistical Methods, Poznań University of Life Sciences, \\ Wojska Polskiego 28, 60-638 Poznań, e-mails: malgorzata.graczyk@ up.poznan.pl, \\ bronislaw.ceranka@up.poznan.pl
}

\section{SUMMARY}

\begin{abstract}
There are many works in the literature on the construction of experimental plans based on weighing designs. Hence, it is useful to compile a catalogue of experimental designs with specific properties. In this work, we investigate the properties of experimental plans constructed using the matrices of spring balance weighing designs. Additionally, an even number of experimental objects is assumed. An overview of the construction methods of these designs and classes of design matrices with selected properties are given. The results make it possible to create a catalogue of experimental designs constructed on the basis of spring balance weighing designs.
\end{abstract}

Keywords: experimental plan, spring balance weighing design

\section{Introduction}

The problem presented in this paper concerns the planning of experiments. In the theory of experiments, which deals with the planning of experiments and, afterwards, analysis and the formulation of conclusions, a significant role is played by the method of experimental planning. Information on experimental material and environmental conditions should be analysed simultaneously with the optimal statistical properties of the considered designs. To obtain the most effective experiment, it is necessary to balance these conditions properly. Increased effectiveness and reduction of the error variance are combined with appropriate assumptions connected with experimental errors and the design. We can consider many factors affecting the value of the variance: some inaccuracy in measurement of the examined characteristic, and in agricultural research, the 
non-homogeneity of experimental designs (for example, non-homogeneous field richness). These properties lead to significant variability in the observations. This can influence the deductions based on the obtained measurements. Hence, we have to plan and arrange the experiment in such a way as to limit the influence of these factors, having regard to the goal of the experiment and the manner of its performance.

We conduct the experiment on the basis of a plan, which is the matrix $\mathbf{X}$, called the design matrix or the design. This matrix describes the arrangement of the experimental units. In this work, we consider spring balance weighing designs. These are designs used to determine unknown measurements of a certain number of objects $p$ with the use of a given number of measurements $n$. The elements of the design matrix $\mathbf{X}$ are zeros or ones, indicating whether or not the selected object is taken into account in subsequent measurements. The result of each measurement is a linear combination of unknown measures of $p$ objects with the coefficients equal to zero or one. The purpose of this research is to determine such a matrix for the experiment plan so that the estimators of unknown measures of objects obtained on the basis of the matrix are the best in terms of a selected criterion.

We consider designs that are D-optimal, i.e. designs for which the determinant of the matrix $\left(\mathbf{X}^{\prime} \mathbf{X}\right)^{-1}$ is minimal. In practice, the product of the variance of the estimators of unknown measures of objects is as small as possible.

This paper is a summary of the results of planning of experiments based on spring balance weighing designs as presented in the literature. We assume that there are no systematic errors and that the measurement errors are uncorrelated and have the same variances.

The purpose of the considerations is to provide classes of spring balance weighing designs together with information about the properties of these designs. The work is intended for those planning experiments, to serve as a guide on how to plan the experiment optimally. 
The research concerns experimental designs in which we determine unknown measures for an even number of objects. Research for an odd number of objects is presented in Graczyk and Ceranka (2019a).

\section{Material and methods}

Let us consider the model of a spring balance weighing design in the form $\mathbf{y}=$ $\mathbf{X w}+\mathbf{e}$, where $\mathbf{y}$ is an $n \times 1$ random vector of observations, $\mathbf{X} \in \boldsymbol{\Phi}_{n \times p}\{0,1\}$, the class of $n \times p$ matrices with elements equal to 0 or 1 , $\mathbf{w}$ is a $p \times 1$ vector of uknown measurements of objects, and $\mathbf{e}$ is an $n \times 1$ random vector of errors. We assume that there are no systematic errors, i.e. $\mathrm{E}(\mathbf{e})=\mathbf{0}_{n}$, and that the errors are uncorrelated and may have the same variances, i.e. $\operatorname{Var}(\mathbf{e})=\sigma^{2} \mathbf{I}_{n}$, where $\mathbf{0}_{n}$ is the $n \times 1$ vector with zero elements everywhere, and $\mathbf{I}_{n}$ is the identity matrix. Several results connected with the properties of estimators of unknown measurements of objects are available in the literature; see Banerjee (1975), Ceranka and Katulska (1987, 1989), Hudelson et al. (1996), Neubauer et al. (1998), Katulska and Przybył (2007), Ceranka and Graczyk (2014, 2017).

Based on Neubauer et al. (1998), the design $\mathbf{X}$ is regular D-optimal in the class $\boldsymbol{\Phi}_{n \times p}\{0,1\}$ for an even number of objects if

$$
\operatorname{det}\left(\mathbf{X}^{\prime} \mathbf{X}\right)=(p+1)\left(\frac{(p+2) n}{4(p+1)}\right)^{p} .
$$

This condition is fulfilled if and only if

$$
\mathbf{X}^{\prime} \mathbf{X}=\frac{(p+2) n}{4(p+1)}\left(\mathbf{I}_{p}+\mathbf{1}_{p} \mathbf{1}_{p}^{\prime}\right)
$$

and each row of the design matrix $\mathbf{X}$ has $0.5 p$ or $0.5(p+2)$ ones. Not for every pair of a number of objects $p$ and a number of measurements $n$ can we determine a matrix that satisfies the optimality conditions.

In the classes $\boldsymbol{\Phi}_{(n-s) \times p}\{0,1\}$ we determine the design $\mathbf{X}_{\gamma}, \gamma=1$, which is D-optimal for $p$ objects and $n-s$ measurements. We add respectively 1, 2, 3 or 4 rows of $\boldsymbol{x}_{s}$-measurements to the design matrix $\mathbf{X}_{\gamma}$. Then we answer the question 
of how to add these next $s$ measurements, $s=1,2,3,4$, so that $\operatorname{det}\left(\mathbf{X}^{\prime} \mathbf{X}\right)^{-1}$ is minimal.

In the papers of Katulska and Przybył (2007) and Ceranka and Graczyk (2014, 2017), the conditions determining the existence of such designs and the methods of their construction were considered. Let $\mathbf{X}_{\gamma}$ be a matrix of a D-optimal spring balance weighing design in the class for $p$ objects and $n-s$ measurements. For the case $s=1$, in the paper of Katulska and Przybył (2007) a spring balance weighing design was considered in the form

$$
\mathbf{X}=\left[\begin{array}{l}
\mathbf{X}_{\gamma} \\
\boldsymbol{x}_{1}^{\prime}
\end{array}\right]
$$

For $s=2$, Katulska and Przybył (2007) considered a spring balance weighing design in the form

$$
\mathbf{X}=\left[\begin{array}{c}
\mathbf{X}_{\gamma} \\
\boldsymbol{x}_{1}^{\prime} \\
\boldsymbol{x}_{2}^{\prime}
\end{array}\right] .
$$

For $s=3$, in Ceranka and Graczyk (2014) the weighing design was considered as

$$
\mathbf{X}=\left[\begin{array}{l}
\mathbf{X}_{\gamma} \\
\boldsymbol{x}_{1}^{\prime} \\
\boldsymbol{x}_{2}^{\prime} \\
\boldsymbol{x}_{3}^{\prime}
\end{array}\right] .
$$

For $s=4$, Ceranka and Graczyk (2017) studied properties of a weighing design in the for

$$
\mathbf{X}=\left[\begin{array}{l}
\mathbf{X}_{\gamma} \\
\boldsymbol{x}_{1}^{\prime} \\
\boldsymbol{x}_{2}^{\prime} \\
\boldsymbol{x}_{3}^{\prime} \\
\boldsymbol{x}_{4}^{\prime}
\end{array}\right] .
$$

For an even number of objects, the design $\mathbf{X}$ in the form $(s)$ is regular D-optimal if and only if 


$$
\mathbf{X}_{\gamma}^{\prime} \mathbf{X}_{\gamma}=\frac{(p+2)(n-s)}{4(p+1)}\left(\mathbf{I}_{p}+\mathbf{1}_{p} \mathbf{1}_{p}^{\prime}\right), \gamma=1, \text { and }
$$

(i) for $\mathrm{s}=1, \boldsymbol{x}_{1}^{\prime} \mathbf{1}_{p}=\frac{p}{2}$ or $\boldsymbol{x}_{1}^{\prime} \mathbf{1}_{p}=\frac{p+2}{2}$

(ii) for $s=2,3,4$ and

a. $\quad p=0(\bmod 4)$, if and only if $\boldsymbol{x}_{h}^{\prime} \boldsymbol{x}_{h^{\prime}}=\frac{p}{4}$ and $\boldsymbol{x}_{\xi}^{\prime} \mathbf{1}_{p}=\boldsymbol{x}_{\varsigma}^{\prime} \mathbf{1}_{p}=$ $\boldsymbol{x}_{\gamma}^{\prime} \mathbf{1}_{p}=\frac{p}{2}, \boldsymbol{x}_{\eta}^{\prime} \mathbf{1}_{p}=\frac{p+2}{2}$, and $\frac{p+2}{2}$ exists ones,

b. $\quad p+2=0(\bmod 4)$, if and only if $\boldsymbol{x}_{h}^{\prime} \boldsymbol{x}_{h^{\prime}}=\frac{p+2}{4}$ and $\boldsymbol{x}_{\xi}^{\prime} \mathbf{1}_{p}=$ $\boldsymbol{x}_{\varsigma}^{\prime} \mathbf{1}_{p}=\boldsymbol{x}_{\gamma}^{\prime} \mathbf{1}_{p}=\frac{p+2}{2}, \boldsymbol{x}_{\eta}^{\prime} \mathbf{1}_{p}=\frac{p}{2}$, and $\frac{\mathrm{p}}{2}$ exists ones,

$h, h^{\prime}=1,2,3,4, h \neq h^{\prime}, h, h^{\prime} \leq s, \xi, \varsigma, \gamma, \eta=1,2,3,4, \xi, \varsigma, \gamma, \eta \leq s, \xi \neq \varsigma \neq \gamma \neq \eta$.

If, on the basis of the above scheme, we are not able to determine the Doptimal design, then we look for the best design and choose one on the basis of the efficiency coefficient. According to the definition given by Bulutoglu (2009), the efficiency of an experimental design is defined as the ratio of the determinant of the information matrix of a given design to the determinant of the information matrix of a potential D-optimal design. In this paper we consider highly Defficient designs, i.e. designs for which $\mathrm{D}_{\text {eff }}^{s}>0.94$. For a matrix $\mathbf{X}$ of the form $(s)$,

$$
\begin{aligned}
& D_{\text {eff }}^{s}= \\
& \frac{n-s}{n}\left\{\begin{array}{ll}
\sqrt[p]{1+\frac{p}{n-s}} & \text { if } s=1 \\
\sqrt[p]{\left(1+\frac{p(p+1)}{(p+2)(n-s)}\right)^{s-1}\left(1+\frac{p(p+s+1)}{(p+2)(n-s)}\right)} & \text { if } s=2,3,4 \text { and } p=0(\bmod 4) \\
\sqrt[p]{\left(1+\frac{p+1}{n-s}\right)^{s-1}\left(1+\frac{p-s+1}{n-s}\right)} & \text { if } s=2,3,4 \text { and } p+2=0(\bmod 4)
\end{array} .\right.
\end{aligned}
$$

Let $\gamma=2$. In the papers of Ceranka and Graczyk (2018,2019b), the conditions determining the existence of highly D-efficient designs and the methods of their construction were considered. Let $\mathbf{X}_{\gamma}$ be a matrix of a highly D-efficient spring balance weighing design in the class $\boldsymbol{\Phi}_{(n-s) \times p}\{0,1\}$ for $p$ objects and $n-s$ measurements. For $\gamma=2$, issues concerning a highly D-efficient spring balance weighing design $\mathbf{X}$ were considered for $s=1,2,3$ in Ceranka and Graczyk (2018), and for $s=4$ in Ceranka and Graczyk (2019b). 
For an even number of objects, the design $\mathbf{X}$ in the form $(s)$ is regular D-optimal if and only if

$$
\mathbf{X}_{\gamma}^{\prime} \mathbf{X}_{\gamma}=\frac{n-s}{4(p-1)}\left(p \mathbf{I}_{p}+(p-2) \mathbf{1}_{p} \mathbf{1}_{p}^{\prime}\right), \gamma=2 \text {, and }
$$

(i) for $\mathrm{s}=1, \boldsymbol{x}_{1}^{\prime} \mathbf{1}_{p}=\frac{p+2}{2}$

(ii) for $s=2,3,4$ and

a. $\quad p=0(\bmod 4)$, if and only if $\boldsymbol{x}_{h}^{\prime} \boldsymbol{x}_{h^{\prime}}=\frac{p+4}{4}$ and $\boldsymbol{x}_{h}^{\prime} \mathbf{1}_{p}=\frac{p+2}{2}$,

b. $p+2=0(\bmod 4)$, if and only if $\boldsymbol{x}_{h}^{\prime} \boldsymbol{x}_{h^{\prime}}=\frac{p+2}{4}$ and, $\boldsymbol{x}_{h}^{\prime} \mathbf{1}_{p}=$ $\frac{p+2}{2}$

$h, h^{\prime}=1,2,3,4, h \neq h^{\prime}$. For such designs

$D_{\text {eff }}^{s}=$

$\frac{p(p+1)(n-s)}{(p-1)(p+2) n}\left\{\begin{array}{ll}\sqrt[p]{\frac{p-1}{p+1}\left(1+\frac{p^{3}+(s-1) p^{2}+8 s}{(n-s) p^{2}}\right)\left(1+\frac{p-1}{n-s}\right)^{s-1}} & \text { if } s=1,2,3,4 \text { and } p=0(\bmod 4) \\ \sqrt[p]{\frac{p-1}{p+1}\left(1+\frac{p^{3}+8}{(n-s) p^{2}}\right)} & \text { if } s=1 \text { and } p+2=0(\bmod 4) \\ \sqrt[p]{\frac{p-1}{p+1}\left(1+\frac{(p+2)\left(p^{2}-(s+1) p+\varphi_{s}\right)}{(n-s) p^{2}}\right)\left(1+\frac{(p-1)(p+2)}{(n-s) p}\right)^{s-1}} & \text { if } s=2,3,4 \text { and } p+2=0(\bmod 4)\end{array}\right.$,

where $\varphi_{2}=81, \varphi_{3}=12, \varphi_{4}=16$.

\section{Results}

Table 1 presents the efficiency coefficients of the designs. If the design is determined on the basis of a D-optimal design, then the efficiency coefficient is written in bold. The remaining coefficients correspond to the construction method of a design matrix based on highly D-efficient designs. The limit of $p \leq 16, n \leq$ 30 was adopted. If the efficiency coefficient is equal to one, then the design in a given class is regular D-optimal. The sign "-" means that for a given number of objects $p$ and a given number of measurements $n$, it is not possible to provide a design construction method based on the approaches presented in this paper. For the given construction methods, $\mathrm{D}_{\text {eff }}$ diagrams for 4 and 6 objects are presented, with the number of measurements limited to 30 . 

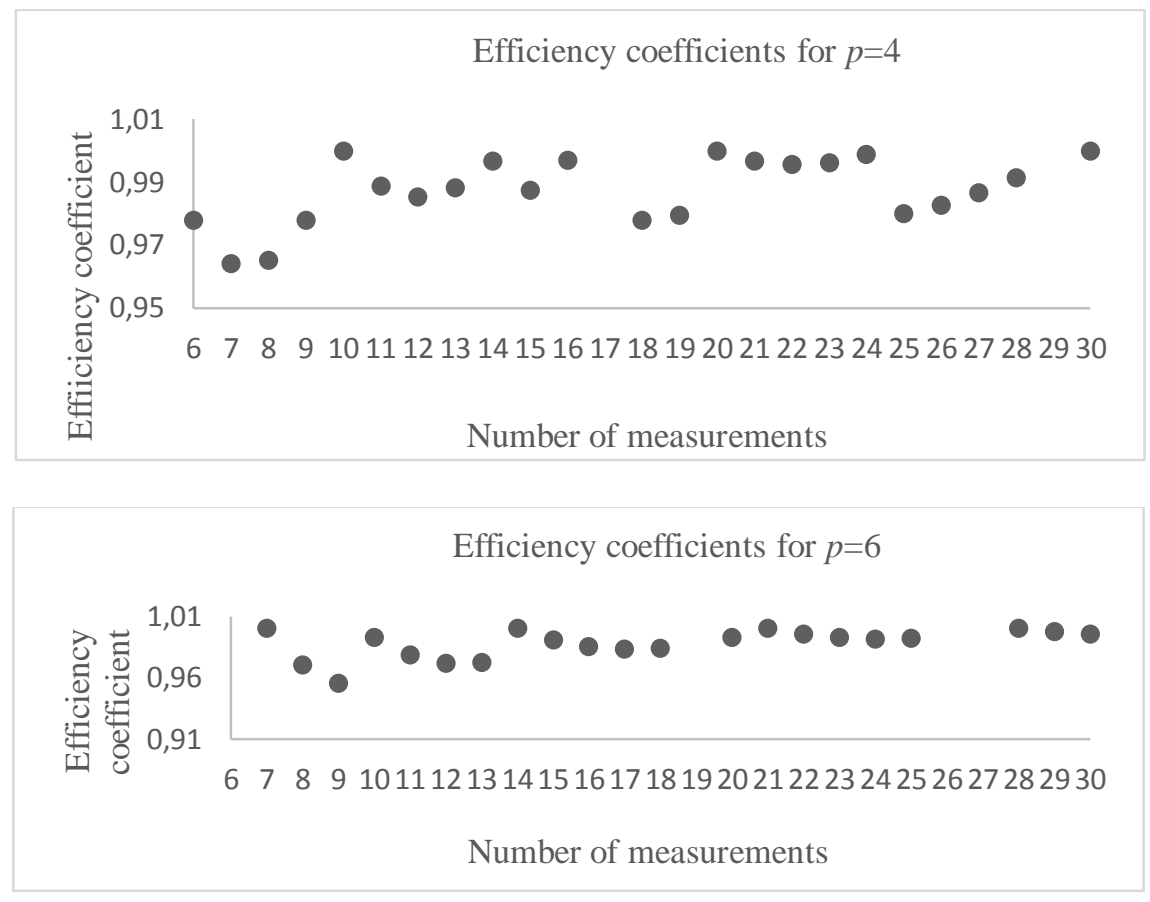

Figure 1. Effectiveness of spring balance weighing designs for a selected number of objects

\section{Discussion}

This paper describes classes of spring balance weighing designs which are D-optimal or highly D-efficient. Unfortunately, not for every pair of a number of objects and a number of measurements can we determine a design with the properties discussed in this paper. However, further possibilities of constructing these designs based on the known matrices of block designs should be considered. It should be noted that among many examples of the use of weighing systems one can find the work of Banerjee (1975) dealing with weighing systems in medicine, economics and operational research. The works of Sloane and Harwit (1976) and Koukouvinos and Seberry (1997) are devoted to planning experiments in optics. 
Table 1. Efficiency coefficients for spring balance weighing designs under the constraints $p \leq 16, n \leq 30$

\begin{tabular}{|c|c|c|c|c|c|c|c|c|}
\hline \multicolumn{9}{|c|}{ Number of objects $p$} \\
\hline & & 4 & 6 & 8 & 10 & 12 & 14 & 16 \\
\hline \multirow{25}{*}{ 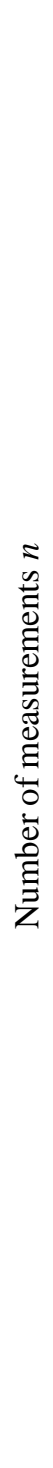 } & 6 & 0.9779 & - & - & - & - & - & - \\
\hline & 7 & 0.9641 & 1 & - & - & - & - & - \\
\hline & 8 & 0.9652 & 0.9701 & - & - & - & - & - \\
\hline & 9 & 0.9779 & 0.9551 & - & - & - & - & - \\
\hline & 10 & 1 & 0.9927 & - & - & - & - & - \\
\hline & 11 & 0.9889 & 0.9783 & - & 1 & - & - & - \\
\hline & 12 & 0.9855 & 0.9719 & - & 0.9779 & - & - & - \\
\hline & 13 & 0.9884 & 0.9723 & - & 0.9628 & - & - & - \\
\hline & 14 & 0.9968 & 1 & 0.9968 & 0.9532 & - & - & - \\
\hline & 15 & 0.9875 & 0.9905 & 0.9851 & 0.9484 & - & 1 & - \\
\hline & 16 & 0.9972 & 0.9851 & 0.9776 & - & - & 0.9827 & - \\
\hline & 17 & - & 0.9830 & 0.9736 & - & - & 0.9694 & - \\
\hline & 18 & 0.9779 & 0.9839 & 1 & 0.9983 & - & 0.9519 & - \\
\hline & 19 & 0.9796 & - & 0.9919 & 0.9887 & - & 0.9450 & - \\
\hline & 20 & 1 & 0.9927 & 0.9866 & 0.9845 & - & - & - \\
\hline & 21 & 0.9968 & 1 & 0.9835 & 0.9776 & - & - & - \\
\hline & 22 & 0.9957 & 0.9954 & 0.9827 & 1 & 0.9990 & - & - \\
\hline & 23 & 0.9964 & 0.9926 & - & 0.9930 & 0.9910 & - & - \\
\hline & 24 & 0.9989 & 0.9915 & - & 0.9879 & 0.9848 & - & - \\
\hline & 25 & 0.9800 & 0.9918 & - & 0.9844 & 0.9803 & - & - \\
\hline & 26 & 0.9829 & - & - & 0.9824 & 1 & 0.9993 & - \\
\hline & 27 & 0.9868 & - & - & - & 0.9939 & 0.9925 & - \\
\hline & 28 & 0.9915 & 1 & 0.9968 & - & 0.9892 & 0.9870 & - \\
\hline & 29 & - & 0.9973 & 0.9935 & - & 0.9857 & 0.9827 & - \\
\hline & 30 & 1 & 0.9956 & 0.9914 & - & 0.9833 & 1 & 0.9996 \\
\hline
\end{tabular}


Among the various applications of weighing systems, the following should be distinguished as relating to agricultural experimentation: Ceranka and Katulska (1987, 1989) and Katulska (1984). Graczyk (2013) describes the possibility of using the weighing design matrix as an experiment plan in plant protection research, and Graczyk (2014) describes an experiment for comparison of the impact of NPK and various types of organic matter applied under the fore crop on the health condition of the stem base successor plant, spring barley. Possible further implementations of the studied systems can be found wherever the experimental plan is based on the plan of the factor designs.

\section{REFERENCES}

Banerjee K.S. (1975): Weighing Designs For Chemistry, Medicine, Economics, Operations Research, Statistics. Marcel Dekker Inc., New York.

Bulutoglu D.A., Ryan K.J. (2009): D-optimal and near D-optimal 2k fractional factorial designs of resolution V. Journal of Statistical Planning and Inference 139: 16-22.

Ceranka B., Graczyk M. (2014): Regular D-optimal spring balance weighing designs: construction. Acta Universitatis Lodziensis, Folia Oeconomica 302: 111-125.

Ceranka B., Graczyk M. (2017): Highly D-efficient weighing design and its construction. Acta Universitatis Lodziensis, Folia Oeconomica 331: 143-151.

Ceranka B., Graczyk M. (2018): Highly D-efficient weighing designs for an even number of objects. Revstat Statistical Journal 16(4): 475-486.

Ceranka B., Graczyk M. (2019a): Katalog planów wybranych układów doświadczalnych. Nauka, Przyroda, Technologie 13(2): 57-64.

Ceranka B., Graczyk M. (2019b): A highly D-efficient spring balance weighing design for an even number of objects. Acta Universitatis Lodziensis, Folia Oeconomica 344: $17-26$.

Ceranka, B., Katulska, K. (1987): Zastosowanie teorii sprężynowych układów wagowych do analizy doświadczeń z mieszankami. Listy Biometryczne XXIV: 17-26.

Ceranka B., Katulska K. (1989): Application of the biased spring balance weighing theory to estimation of differences of line effects for legume content. Biometrical Journal 31: 103-110.

Graczyk M. (2013): Some applications on weighing designs. Biometrical Letters 50: $15-26$.

Graczyk M. (2014): Relations between randomized block designs and weighing designs in examples. Colloquium Biometricum 44: 97-107.

Hudelson M., Klee V., Larman D. (1996): Largest j-simplices in d-cubes: Some relatives to the Hadamard determinant problem. Linear Algebra and its Applications 24: 519-598. 
Katulska K. (1984): Zastosowanie teorii układów wagowych do badania upraw paszowych i w geodezji. Czternaste Colloquium Metodologiczne z Agro-Biometrii, PAN: 195-208.

Katulska K., Przybył K. (2007): On certain D-optimal spring balance weighing designs. Journal of Statistical Theory and Practice 1: 393-404.

Koukouvinos C., Seberry J. (1997): Weighing matrices and their applications. Journal of Statistical Planning and Inference 62: 91-101.

Neubauer G.N., Watkins W., Zeitlin J. (1998): Notes on D-optimal designs. Linear Algebra and its Applications 280: 109-127.

Sloane N.J.A., Harwit M. (1976): Masks for Hadamard transform optics, and weighing designs. Applied Optics 5: 107-114. 